\title{
2020 Young Researcher Forum for 15th Global Summit on Oncology and Cancer
}

\author{
Stefan Glueck \\ Vice President, Oncology, Celgene Corporation, USA, E-mail: sgluck@ celgene.com
}

We take huge pride to announce 15th Global Oncology and Cancer which is slated to hold from 18-19 March, 2020 at Frankfurt, Germany.

Oncology 2020 YFR, provides a novel platform for Young Researchers/Investigators for presenting recent researches with an in-intensity analysis. Oncology 2020 Conference cordially invites Young from Universities/Institutes/Industries to present an oral or poster presentation. The Young Researchers Forum provides an opportunity to the budding and young researchers to meet and discuss research topics and approaches to share and develop ideas, acquire from one another and advance knowledge from high-ranking researchers.

All the talented Young researchers, scientists, Post-doctoral fellows, Young Investigators, Post-Graduate students, Trainees, Junior faculty will be awarded in the acknowledgment of their outstanding contribution towards the conference. The Young Researcher Award takes every effort in providing a solid development opportunity for initial career academicians by meeting professionals to discuss and share their experiences on all aspects of Oncology and Cancer.

The best YRF award is to identify the notable work done by the student participants in our Oncology 2020 Conference. The award is announced revising the excellence and contribution done towards the conference and to the global scientific community. In addition, considering the outstanding, inspiring, effective and professional presentation which engaged all the attendees of our Conference? Also this is especially for the young or budding researcher who has achieved a greater milestone in the field of Oncology and Cancer.

The conference is centred and focused to the theme of "Ray of Hope on Bridging Cancer Research and Treatment". It gives an awesome stage to cover recent breakthroughs in $\underline{\text { Cancer }}$ Biology, Oncology Causes and Diagnosis, Preventive and Translational Oncology, Medical and Radiation Oncology, Biomarkers, Lung Cancer and Breast Cancer. The conference will create a matchless experience for the participants.

\section{Benefits of Oncology 2020 YRF:}

- Showcase your recent research by oral presentations.

- Learn about profession development and the latest research gear and technologies in your field.

- This discussion board will supply pertinent and timely details to the individuals who conducts studies and those who use and benefit from research.

- Develop a foundation for collaboration among younger researchers.

- The forum will provide an opportunity for collegial interaction with other young investigators and established senior investigators throughout the globe.
- Interact and share thoughts with both peers and mentors.

- Opportunity for young researchers to learn about the research regions in their peers to grow their capability as multidisciplinary researchers.

\section{Eligibility:}

Young researchers should be Post-Graduate students, Postdoctoral fellows, Trainees, Junior faculties are the focus point for this event. The topic must fit into scientific tracks of the conference. Each individual participant is authorized to submit their one paper (as first author or co-author). None of the authors must have senior position. Participants must be below the age of 33 years. All submissions need to be in English. Opportunity for young researchers to study the research area of their colleagues to increase their potential as multidisciplinary researchers.

To know more about the conference: https://oncologysummit.alliedacademies.com/young-researchers-forum

\section{Contact Person:}

David Thomas

Program Manager | Oncology 2020

Email: mailto:oncology@tumorconferences.com

WhatsApp: +44-1258650017 